\title{
Paced Ventricular Aggregate QRS Duration
}

National Cancer Institute

\section{Source}

National Cancer Institute. Paced Ventricular Aggregate QRS Duration. NCI Thesaurus.

Code C117781.

An aggregate paced QRS duration value based on the measurement of paced QRS duration intervals from multiple beats within a single ECG. The method of aggregation, which can vary, is typically a measure of central tendency such as the mean. (CDISC) 